\title{
Migrationsbedingte Mehrsprachigkeit in Zeiten von Corona und darüber hinaus - Herausforderungen und Chancen im Hinblick auf die Homeschooling-Problematik. Vorüberle- gungen zu einem empirischen Forschungs- und Entwicklungsprojekt
}

\author{
Andrea Bambek - Csaba Földes - Laura Kuhlig
}

„Never let a good crisis go to waste."

(Winston Churchill)

Migration-related multilingualism in times of Corona and beyond - challenges and opportunities with regard to the homeschooling problem. Preliminary considerations for an empirical research and development project

Abstract

This paper exemplifies some preliminary considerations of a current conceptualised project that focuses on a research and a development component in the fields of German applied linguistics and didactics of German as a second language by exploring the effects of the corona pandemic situation on learning and teaching under migration-induced multilingualism. The project concept, its content design and objectives are presented and placed in the general context of migration-related multilingualism in the German education system. The proposed project also intends to support research output beyond the pandemic and provide sustainable teaching and learning materials.

Keywords: multilingualism; migration and educational opportunities; German as a second language; home schooling; material development for language-sensitive teaching

Schlüsselwörter: Mehrsprachigkeit; Migration und Bildungschancen; Deutsch als Zweitsprache; Homeschooling; Materialentwicklung für sprachsensiblen Unterricht

Subject-Affiliation in New CEEOL: Language and Literature - Applied Linguistics Sociolinguistics

DOI: 10.36007/eruedu.2020.3.005-017 


\section{Einleitung, Problemstellung und Zielsetzung}

Die aktuelle Corona-Pandemie bereitet nicht nur für das Gesundheitswesen, sondern natürlich auch für die Politik, die Wirtschaft, für Bildung und Ausbildung sowie für sämtliche Bereiche des gesellschaftlichen Lebens eine besondere, in dieser Form in der jüngsten Geschichte noch nicht dagewesene Herausforderung. Alltagserfahrungen zeigen: Die Einstellung des Präsenzbetriebs an Schulen und der darauffolgende Unterricht im „Homeschooling“ (auch Heimunterricht) bedeutet für Lehrkräfte, Schüler(innen) sowie Eltern zunehmend Probleme und Überforderung. Schulunterricht unter Pandemiebedingungen belastet selbst Akademikerhaushalte und andere bildungsaffine Bevölkerungsschichten. Für Schüler(innen) mit Migrationshintergrund potenziert sich das Problem aus mehreren Gründen: Bedingt durch ihre Migrationsgeschichte liegen bei innen zum Teil andere Lern- und Digitalkulturen vor, und sie sind oft auf Förderunterricht und auf einen sprachsensiblen Fachunterricht, der sie gleichermaßen sprachlich und fachlich fördert, angewiesen. Ebenso können Eltern mit Migrationshintergrund wegen nicht hinreichender deutscher Sprachkompetenz häufig ihre Kinder ohne professionelle Hilfe nicht unterstützen. Gleichwohl sind Lehrkräfte für den qualifizierten Umgang für einen differenzierten und fördernden Unterricht unter Bedingungen sprachlicher und kultureller Heterogenität (auch in Nicht-Corona-Zeiten) vielerorts nur ungenügend qualifiziert.

Vor diesem Hintergrund wird im Beitrag ein aktuell konzipiertes Projekt der Verfasserinnen und des Verfassers im Hinblick auf seine Anlage, Zielstruktur und inhaltliche Matrix vorgestellt und in den allgemeinen Kontext der migrationsbedingten Mehrsprachigkeit im deutschen Bildungssystem gesetzt. Ein zentrales Ziel des Projekts ist es, durch empirische Forschung die gegenwärtigen Problembereiche und Herausforderungen der pandemiebedingten Schulsituation für Lehrer(innen) und Schüler(innen) zu eruieren. Ein weiteres Ziel besteht darin, durch Entwicklung von gezielten Lehr- und Lernformaten sowie von Lernmaterialien einer Zunahme von Bildungsdefizit bei Schüler(inne)n mit Migrationshintergrund entgegenzuwirken. Der Begriff „Homeschooling“ wird in der Corona-Pandemie meist unreflektiert verwendet, obwohl „Homeschooling“ als Terminus und die damit bezeichneten Bereiche seit Längerem Objekte wissenschaftlicher Forschungen und entsprechender Diskurse sind. Deshalb wird zunächst der Kontexthintergrund „Homeschooling“ als Komplexphänomen beleuchtet. Wie bereits oben angedeutet, trifft sprachliche und kulturelle Heterogenität Lehrer(innen) in Deutschland oft unvorbereitet, da die Lehrerbildung - immer noch überwiegend - auf monolingual deutsche Klassen ausgerichtet ist und die faktisch vorhandene Mehrsprachigkeit in deutschen Schulen nicht genügend berücksichtigt. Zur Skizzierung der Rahmenparameter werden in Abschnitt 3 Aspekte der Lehrerbildung im Hinblick auf Deutsch als Zweitsprache $(\mathrm{DaZ})^{1}$ sowohl im deutschlandweiten Kontext als auch bezogen auf die Universität

\footnotetext{
1 Deutsch als Zweitsprache als Spracherwerbsprozess wird - idealtypisch - definiert als (überwiegend) ungesteuerter Spracherwerb, der meist ab dem dritten Lebensjahr, nach dem Erstspracherwerb im Zielland (Deutschland) erfolgt und wird von Deutsch als Fremdsprache, als gesteuertem Sprachlernen, das im Ausland stattfindet, abgegrenzt. Im schulischen Kontext ist überwiegend von $\mathrm{DaZ}$ die Rede, obwohl Schüler(innen) in Bildungseinrichtungen in gezielten Förderprogrammen
} 
Erfurt (die Wirkungsstätte der Verfasserinnen und des Verfassers) beleuchtet. Abschließend erfolgt eine Erörterung des Projektkonzepts und des Methodendesigns unter Einbeziehung angestrebter Synergieeffekte mit den aktuell laufenden Forschungsprojekten und Forschungskooperationen an der Universität Erfurt.

\section{Kontexthintergrund Homeschooling}

In Deutschland nimmt die pandemiebedingte Verlagerung des Unterrichtsgeschehens aus der Schule in die häusliche Umgebung eine Sonderstellung zum klassischen Homeschooling ${ }^{2}$ ein. Um eine genaue Abgrenzung herausarbeiten zu können, soll zunächst das traditionelle Konzept des Heimunterrichts beleuchtet werden. Dazu wird neben der amerikanischen Idee dieses Bildungsweges und der Begriffsdiskussion auch die Homeschool-Situation in Deutschland eruiert.

Im Gegensatz zur Notwendigkeit des häuslichen Unterrichts im besiedelten Nordamerika Anfang des 17. Jahrhunderts infolge eines noch nicht vorhandenen Schulsystems, setzte sich die Homeschool-Bewegung ${ }^{3}$, die in den 1960er ihren Anfang fand, für eine gezielte Alternative zum institutionalisierten Unterricht an Schulen ein (Gaither 2017, 6ff. und 93). Durch die Legalisierung von Homeschooling in den 80er und frühen 90er Jahren des 20. Jahrhunderts in allen Bundesstaaten der USA (siehe Gaither 2017, 207) entstanden verschiedene Bezeichnungen für das Lehren und Lernen im häuslichen Umfeld.

Inhaltlich stellt Brabant $(2008,297)$ fest: „Home schooling can be defined as the education of school-aged children under their parents' supervision, in and around the home, and in place of full-time school attendance“. Diese Merkmale sind nach Brabant $(2008,297)$ die einzigen klaren Parameter zur Bestimmung dieser Bildungsform, die ansonsten in Bezug auf Lehr- und Lerneinstellungen, Lernorte und soziale Interaktionen sehr unterschiedlich ausgeübt wird. Homeschooling oder Home-Based Education umfasst eine funktionsfähige alternative Form der Schulbildung, bei der Eltern/Erziehungsberechtigte die Hauptverantwortung für die Erziehung und Bildung ihrer Kinder zu Hause übernehmen, anstatt diese öffentlichen oder privaten Schuleinrichtungen zu überlassen (Martin-Chang-Levesque 2017, 121). Während sich der Terminus „Schooling“ an der klassischen Bildungsform Schule anlehnt, umfasst „Education“ eine weitaus größere Palette an Motiven sowie Lehr- und Lernformen. Auch wenn es zahlreiche deutsche Begrifflichkeiten für das komplexe Konzept gibt, wie beispielsweise Spiegler $(2008,12)$ auflistet: „Hausunterricht, Heimschule, Heimunterricht, Familienschule, Bildung zu Hause, häuslicher Unterricht und Schule zu Hause“, wird auch im deutschsprachigen Raum in der Regel auf die englischen Termini zurückgegriffen. „Homeschoo-

durchaus gesteuert (im Sinne von Deutsch als Fremdsprache) Deutsch lernen. Eine trennscharfe Unterscheidung zwischen Deutsch als Zweit- und Fremdsprache ist oft nicht möglich. Zur Diskussion um weitere Definitionsaspekte vgl. Ahrenholz (2017). DaZ in der Lehrerausbildung meint, dass angehende Lehrer(innen), und zwar aller Fächer, Ausbildungselemente u.a. in den Bereichen Zweitspracherwerb, Didaktik und Methodik des Zweitsprachunterrichts, interkulturelle Aspekte u.v.m. absolvieren. Vgl. hierzu Abschnitt 3.

2 Auch in der Form ,home schooling“ oder „,home-schooling“ bekannt.

3 Für eine detaillierte historische Zusammenfassung des Homeschoolings in den Vereinigten Staaten siehe Gaither (2017). 
ling“ und „Home Education“ werden mitunter synonym gebraucht, wobei sich im wissenschaftlichen Diskurs, wie Spiegler (2018, 11f.) nachweist, die Bezeichnung „Home Education“ durchgesetzt hat. Unter „Home Education“ versteht man „den Bildungsansatz, bei dem Kinder in ihrem eigenen häuslichen Umfeld lernen, anstatt eine Schule zu besuchen. Gestaltet, organisiert oder begleitet wird dieser Lernprozess meist durch die Eltern" (Spiegler 2008, 11). ${ }^{4}$ Aus erziehungswissenschaftlicher Perspektive arbeitet Ladenthin (2018, 519ff.) vier Typen heraus: (a) lebensweltliches Homeschooling, ${ }^{5}$ (b) ständisches Homeschooling, ${ }^{6}$ (c) modernes Homeschooling und (d) additives Homeschooling. Mit Blick auf das Anliegen des vorliegenden Aufsatzes sollen nun die Typen (c) und (d) näher interpretiert werden. Das moderne Homeschooling ist als aktive Gegenbewegung zur entstandenen Schulpflicht anzusehen, dessen Entstehung auf dem „Prinzipienkonflik[t] zwischen dem staatlichen Auftrag der Existenzsicherung und dem Recht auf individuelle Freiheit" (Ladenthin 2018, 520) zurückzuführen ist. Diese individuelle Freiheit soll sich in der elterlichen Bestimmung des Bildungsweges ihrer Kinder widerspiegeln. Im Gegensatz dazu wird beim additiven Homeschooling der Schulbesuch durch zusätzliche Angebote und Hilfen der Eltern oder eines Nachhilfelehrers erweitert und ergänzt. Hierzu gehören neben der Hausaufgabenkontrolle und der Erarbeitung des Lernstoffes auch „die ausschließlich von Eltern initiierten, kontrollierten und finanzierten Bildungsprozesse in Sportvereinen und Musikschulen" (Ladenthin 2018, 521).

Die deutsche Homeschool-Bewegung, deren Anfänge in der Mitte des 20. Jahrhunderts zu verorten sind (Spiegler 2012, 56), wird vor allem durch die gesetzlichen Gegebenheiten ${ }^{7}$ in Deutschland vor enorme Hindernisse gestellt. Die Pflicht zum Schulbesuch besteht in allen Bundesländern (Blok-Karsten 2011, 146). Nichtsdestotrotz werden nach Blok-Karsten $(2011,146)$ schätzungsweise 500 Kinder im häuslichen Umfeld unterrichtet, da sich deren Eltern den gesetzlichen Regelungen widersetzen. Da für Homeschooling in Deutschland keine Regularien bestehen, ,liegen nur wenige systematische Studien vor [...], es fehlt an einer umfassenden Rezeption der nichtdeutschen Literatur [...]; es fehlt an quantitativen und qualitativen Untersuchungen über die Bildungschancen und Bildungswege von Homeschool-Kindern“, beklagt Ladenthin (2018, 523). Spiegler (2008) beleuchtet als Erster die elterlichen Gründe für Home Education sowie die familiären Lebenssituationen und untersucht die methodische Gestaltung des Homeschoolings in Deutschland. Reliable Daten über Ergebnisse und Bildungserfolge von Homeschool-Kindern liegen in Deutschland aufgrund fehlender Erhebungen nicht vor. Spiegler $(2012,59)$ verweist allerdings auf punktuelle Fallbeispiele ${ }^{8}$ in denen

4 Der häusliche Unterricht zeichnet sich zudem dadurch aus, dass er „,bildungsorientier[t], systematisc[h] und qualitativ beurteilba[r]" (Ladenthin 2018, 519) ist.

5 Die Weitergabe von Kulturtechniken, Alltagsritualen und von lebensweltlichem Handeln an die nächste Generation, siehe Ladenthin (2018, 519f.).

6 Abhängig von der jeweiligen gesellschaftlichen Stellung der Eltern wurden verschiedene ,ständische“ Motive im Homeschooling identifiziert, siehe Ladenthin $(2018,520)$.

7 Ausführliche Informationen zur juristischen Debatte in Deutschland und illustrative Einzelfallbeispiele findet man bei Reimer (2012) und Spiegler (2008).

8 Mehr Informationen zu den vorhandenen Ergebnissen der Einzelfallbeispiele siehe Spiegler (2008, 127ff.). 
Homeschooler positiv in Leistungstests abschnitten oder sich erfolgreich in das deutsche Schulsystem integrierten.

Zusammenfassend ist festzustellen, dass Homeschooling bzw. Home Education im klassischen Sinn eine bewusste Entscheidung gegen das öffentliche Schulsystem und für eine aktive Ausgestaltung der schulischen Bildung der eigenen Kinder durch die Eltern im häuslichen Umfeld ist. Für die coronabedingte temporäre Aussetzung des Präsenzbetriebs an deutschen Schulen kann die obige Definition nicht angewandt werden, vielmehr lässt sich die Situation des eingeschränkten Schulbetriebs als eine Erweiterung des additiven Homeschoolings von Ladenthin (2018) begreifen. Das Verständnis von Homeschooling in unserem Projektkonzept ist vor dem Hintergrund der aktuellen Situation ein von Lehrer(inne)n organisierter und durch Eltern begleiteter Unterricht zu Hause. Dabei übernehmen die Lehrpersonen weiterhin die konzeptuelle Gestaltung des Unterrichts, in dem sie den Schüler(inne)n Übungen und Aufgaben zur Verfügung stellen und damit den Arbeitsumfang für das jeweilige Schulfach klar vorgeben. Die Verantwortung der Eltern besteht darin, wie beim additiven Homeschooling, das Monitoring der Aufgabenerledigung zu übernehmen sowie adäquate Förderungsoptionen anzubieten.

\section{Kontexthintergrund DaZ und sprachliche Bildung in der Leh- rerbildung}

Angehende und praktizierende Lehrer(innen) stehen in Deutschland - nicht erst seit den großen Migrationsbewegungen im Jahre 2015 - zunehmend vor mehrsprachigen und kulturell heterogenen Klassen. Von Seiten der einschlägigen DaZ-Forschung werden deshalb schon seit Längerem Ausbildungsinhalte in DaZ für die Lehrerbildung gefordert (vgl. hierzu exemplarisch Steinmüller 1984, Rösch 2009 und Baumann 2017). Insgesamt stellt sich die Lage in Deutschland hinsichtlich der Ausbildung von Lehrkräften in den Bereichen DaZ und sprachliche Bildung ${ }^{9}$ als sehr heterogen dar: Die Spannbreite reicht von einigen wenigen Bundesländern, in denen solche Ausbildungselemente im Lehrerbildungsgesetz verankert sind (wie z.B. Nordrhein-Westfalen ${ }^{10}$ und Berlin ${ }^{11}$ ) bis zu Bundesländern, die solche Inhalte erst momentan zu etablieren versuchen. ${ }^{12}$ Bei der Entwicklung und Implementierung von sog. DaZ- oder Sprachbildungsmodulen stellen sich mehrere Fragen, die in verschiedenen Forschungsansätzen aktuell erforscht werden. Zu innen gehören beispielsweise: Ist ein eigenständiges Modul im Rahmen der Lehrerbildung oder eben eine querschnittliche Verankerung der Inhalte im gesamten Studium zielführender? Welche Inhalte sollen dann die Module haben?

9 Die Bezeichnung „,sprachliche Bildung“ zielt darauf ab, dass nicht nur DaZ-Lernende Sprachförderung benötigen, sondern alle Schüler(innen), die sprachliche Schwierigkeiten im Unterricht haben.

10 In Nordrhein-Westfalen ist seit 2009 im Lehrerbildungsgesetz (Lehrerbildungsgesetz vom 12. Mai 2009 Nordrhein-Westfalen, LAB) festgelegt, dass Lehramtsstudierende ein Modul „Deutsch für Schülerinnen und Schüler mit Zuwanderungsgeschichte" absolvieren müssen.

11 Der Studienanteil „Sprachförderung mit Deutsch als Zweitsprache“ seit 2014 Teil des Berliner Lehrkräftebildungsgesetzes (§ 1 Abs. 2 LBiG; zur Entwicklung der Berliner DaZ- und Sprachbildungsmodule vgl. Börsel-Lütke 2017).

12 Eine diesbezügliche Zusammenschau findet sich bei z.B. Becker-Mrotzek-Rosenberg-SchroederWitte (2017). 
Wie lässt sich die DaZ-Kompetenz von Lehramtsstudierenden modellieren und messen? Auch das Thema „DaZ und Sprachbildung in allen Fächern“ und die Entwicklung von sprachsensiblen Unterrichtsmaterialien für alle Fächer haben zurzeit Hochkonjunktur, vgl. etwa Benholz-Frank-Gürsoy (2015). Das bis dato einzige Kompetenzmodell, das fachunterrichtsrelevante DaZ-Kompetenzen für angehende Lehrkräfte berücksichtigt, stammt von Carlson-Ehmke-Fischer-Hammer-KökerOhm-Rosenbock-Agyei (2018). Die Unterschiede hinsichtlich DaZ und Sprachbildung in der Lehrerbildung in den einzelnen deutschen Bundesländern gründen sich nicht zuletzt auf unterschiedliche bildungspolitische und institutionelle Rahmenbedingungen. So hängt beispielsweise die Frage, ob Ausbildungsinhalte aus dem Bereich DaZ für alle Lehramtsstudierende aller Fächer verpflichtend sind, vom Lehrerbildungsgesetz des jeweiligen Bundeslandes ab. Außerdem gibt es zahlreiche (oft vage formulierte) Empfehlungen und Richtlinien bezüglich Sprachförderung und sprachlicher Heterogenität in den jeweiligen Rahmenvereinbarungen zwischen den zuständigen Kultusministerien und Universitäten. Hinzu kommt noch, dass jede Universität ihre ,eigene Lehrerbildung“ und damit auch unterschiedliche Implementierungsmöglichkeiten von DaZ-Inhalten ins Lehramtsstudium hat. So erfolgt beispielsweise die universitäre Lehrerbildung im Bundesland Thüringen an den Universitäten Jena und Erfurt in zwei unterschiedlichen Systemen. In Jena ist die Ausbildung einphasig, die Lehramtskandidat(inn)en legen folglich Staatsexamina ab, in Erfurt hingegen studieren sie Lehramt im Bachelor-Master-System. An der Universität Erfurt sind Ausbildungsinhalte in DaZ noch keine obligatorischen Anteile der Lehrerbildung. Außerdem kommt erschwerend hinzu, dass Thüringen das einzige deutsche Bundesland ohne herkunftssprachlichen Unterricht ist. Um in dieser Situation Ausbildungsinhalte zu DaZ und Sprachbildung an der Universität Erfurt auf der Ebene der Forschung und der Lehrerbildung nachhaltig zu verankern, sind die Verfasserinnen und der Verfasser in ihrem bereits laufenden Projekt „Sprachliche Bildung in mehrsprachigen Kontexten“ forschend tätig (hierzu ausführlicher unter 4.3).

\section{Migrationsbedingte Mehrsprachigkeit unter Bedingungen der Corona-Pandemie und darüber hinaus - Skizze eines For- schungs- und Entwicklungsprojekts}

\subsection{Projekthintergrund, Anliegen und Fragestellungen}

Viele internationale Bildungsstudien - wie z.B. PISA - belegen, dass in Deutschland Kinder und Jugendliche mit Migrationshintergrund in vielen Bereichen schlechter abschneiden als ihre Mitschüler(innen) ohne Migrationshintergrund. Aus dem Bericht des Jahres 2018 ist beispielsweise ersichtlich, dass Schüler(innen) mit Migrationshintergrund in der Lesekompetenz 63 Punkte hinter Kindern ohne Migrationshintergrund liegen. ${ }^{13}$ Diese grundsätzlich bereits seit Längerem bundesweit bestehenden Unterschiede zuungunsten von Migrantenkindern werden durch

$13 \mathrm{Zu}$ den Ergebnissen der PISA-Studie hinsichtlich Deutschland vgl. https://www.oecd.org/berlin/ themen/pisa-studie/PISA2018_CN_DEU_German.pdf. Abgerufen am 08.07.2020. 
die Corona-Krise vielerorts, so auch in Thüringen, offenbar verschärft, weil derzeit etwa wegen Homeschooling zusätzliche Förderangebote nicht realisiert werden können und der tägliche Kontakt zu deutschsprachigen Lehrkräften und Mitschüler(inne)n fehlen. Wie die Pandemiesituation sich auf das Lehren und Lernen in mehrsprachigen und kulturell heterogenen Klassen genau auswirkt, ist noch nicht erforscht: Die Problematik nimmt in der aktuellen Debatte nur eine marginale Rolle ein, obwohl sie den gesellschaftlich und bildungspolitisch sehr wichtigen Bereich der Bildungsgerechtigkeit betrifft. So thematisieren z.B. die zur pandemiebedingten Situation bereits durchgeführten Studien ${ }^{14}$ die Lage von Schüler(innen) mit Migrationshintergrund überhaupt nicht. Die Mannheimer Corona-Studie fokussiert z.B. auf verschiedene gesellschaftlich relevante Themen wie etwa: berufliche Situation (Homeoffice, Jobverlust), finanzielle Engpässe, Zufriedenheit mit dem Krisenmanagement einzelner Politiker, soziale Kontakte, persönliche Betroffenheit mit dem Virus, Ängste und mehr. ${ }^{15}$ Zwar wird auch „die Betreuungssituation von Kindern und Jugendlichen unter 16 Jahren vor und während der Corona-Krise" als ein Themenschwerpunkt behandelt, aber wie der Titel schon signalisiert, geht es hierbei eher um die Frage, ob Eltern oder eine fremde Person die Kinder beaufsichtigen und ob es z.B. ein ausgeglichenes Betreuungsverhältnis zwischen Vätern und Müttern gibt. ${ }^{16}$ Zwei weitere Studien, jeweils an der Otto-von-Guericke-Universität in Magdeburg ${ }^{17}$ und der Universität Koblenz-Landau ${ }^{18}$ durchgeführt, konzentrieren sich auf die elterliche Sicht auf die häusliche Unterrichtsperiode. Die Magdeburger Erziehungswissenschaftlerin Raphaela Porsch untersucht in ihrer bundesweiten Onlinebefragung unter Eltern von Grundschulkindern neben den Folgen und Belastungen von Homeschooling auch die Aufgabenverteilung in den einzelnen Unterrichtsfächern sowie die Kommunikationswege zwischen Eltern und Lehrpersonen sowie zusätzliche Unterstützungsangebote der Lehrkräfte. ${ }^{19}$ Die Studie „HOMEschooling 2020“, geleitet von der Erziehungswissenschaftlerin Anja Wildemann und dem Psychologen Ingmar Hosenfeld an der Universität Koblenz-Landau, deckt neben dem Primar- auch den Sekundarstufenbereich ab. Die Erhebung erfasst die Themenkomplexe „Organisation zu Hause, Organisation von der Schule, Aufgaben, Unterstützung, Rückmeldung/Feedback, Motivation und Belastung/Akzeptanz" (Wildemann-Hosenfeld 2020, 4). Die demographischen Daten legen dar, dass größtenteils Eltern mit einem mittleren oder höheren Bildungsabschluss an der Befragung teilgenommen haben. Lediglich knapp 3\% der Befragten geben einen Hauptschulabschluss als höchsten Bildungsabschluss an,

$14 \mathrm{Vgl}$. etwa die Studie der Universität Mannheim unter https://www.uni-mannheim.de/gip/corona-studie/ oder die Studie der Robert Bosch Stiftung in Zusammenarbeit mit der Wochenzeitung DIE ZEIT unter https://deutsches-schulportal.de/unterricht/das-deutsche-schulbarometer-spezial-corona-krise/. Abgerufen am 08.07.2020.

15 Vgl. https://www.uni-mannheim.de/gip/corona-studie/\#c187010. Abgerufen am 09.07.2020.

16 Vgl. https://www.uni-mannheim.de/media/Einrichtungen/gip/Corona_Studie/2020-04-05_

Schwerpunktbericht_Erwerbstaetigkeit_und_Kinderbetreuung.pdf. Abgerufen am 09.07.2020.

17 Vgl. https://www.volksstimme.de/lokal/magdeburg/homeschooling-magdeburger-professorin-befragt-eltern. Abgerufen am 08.07.2020.

18 Vgl. https://www.uni-koblenz-landau.de/de/landau/fb5/bildung-kind-jugend/grupaed/medienordner-grundschulpaedagogik/Wildemann/bericht_homeschooling. Abgerufen am 07.07.2020.

19 Siehe https://www.volksstimme.de/lokal/magdeburg/homeschooling-magdeburger-professorin-befragt-eltern. Abgerufen am 07.08.2020. 
nur $0,2 \%$ der Umfrageteilnehmenden besitzen keinen Bildungsabschluss. Somit sind bildungsschwache Familien in der Studie unterrepräsentiert, sodass die Situation und Probleme dieser Gruppe keine Berücksichtigung finden.

Wie aus der obigen Darstellung hervorgeht, sind migrationsbedingte Mehrsprachigkeit und damit verbundene Herausforderungen kein explizites Anliegen der Studien. Unser Projekt soll hingegen - mit Schwerpunkt auf unterrichtliche Aspekte zwischen Schüler(inne)n und Lehrer(inne)n in mehrsprachigen Klassen - sowohl eine Forschungs- als auch eine Entwicklungskomponente auf dem Feld der germanistischen angewandten Linguistik sowie der DaZ-Didaktik umfassen und verfolgt folgende Ziele:

(1) Eine evidenzbasierte Erforschung von Auswirkungen der Corona-Pandemie auf Lernen und Lehren unter migrationsbedingter Mehrsprachigkeit: Im ersten Projektabschnitt werden konkrete Herausforderungen, Bedarfe und Bedürfnisse seitens von Lehrer(inne)n und Schüler(inne)n im Zusammenhang mit Homeschooling (und eines eventuell darauffolgenden „reduzierten Schulbetriebs") empirisch erhoben.

(2) Auf (1) basierend zielt der zweite Projektabschnitt auf eine Ausarbeitung und Bereitstellung von Unterstützungstools für Lehrer(innen) und Schüler(innen) ab. Zu ihnen gehören:

a) Erstellung von (überwiegend) digitalen Formaten (wie z.B. Blended-Learning) zur fachlichen Weiterbildung und zum Coaching von Lehrer(inne)n zum qualifizierten Umgang mit sprachlicher Heterogenität in Zeiten von Corona und darüber hinaus.

b) Entwicklung von Lehr- Lernmaterialien zum sprachsensiblen Unterricht, Erstellung einer Datenbank, Einspeisung der Materialien, die im Internet kostenfrei und langfristig digital zur Verfügung stehen werden.

(3) Als dritter Projektabschnitt werden die von uns entwickelten Lehrformate und Materialien durch empirische Evaluationen (z.B. Befragungen) auf ihre Wirksamkeit und Passgenauigkeit geprüft. Auch wenn dieser Schritt hier als dritte (und forschungslogisch letzte) Phase bezeichnet wird, wird er als ein permanenter zirkulärer Prozess im Sinne der Qualitätssicherung verstanden. Hierdurch soll auch gewährleistet werden, dass bestimmte Formate möglichst schnell eingesetzt werden können.

\subsection{Forschungsdesign und Methoden}

Zur konkreten Bedarfsermittlung für den Unterricht in heterogenen Klassen und zur Evaluation der entwickelten Lehr- und Lernformate wird methodisch der Ansatz der Methodentriangulation gewählt. ${ }^{20} \mathrm{Im}$ Projekt kommen qualitative und quantitative Befragungen zum Einsatz. Unser Vorhaben vertritt die Position, dass qualitative und quantitative Instrumente zu Paradigmen gehören, die sich nicht gegenseitig ausschließen. Vielmehr werden sie als Forschungswerkzeuge gegenstandsangemessen in verschiedenen Phasen des Forschungsprojekts eingesetzt. Im ersten explorativen Abschnitt werden Schüler(innen) mit Migrationshintergrund sowie

20 Über deren Forschungsaspekte, ihre Diskussion und Einsatzmöglichkeiten siehe z.B. Aguado (2015). 
Lehrer(innen) verschiedener Schultypen und verschiedener Unterrichtsfächer zu ihren Erfahrungen in der zurückliegenden coronabedingten Homeschooling-Zeit und der darauffolgenden „kontaktreduzierten“ Unterrichtsphase befragt. Die Befragungen erfolgen in unterschiedlichen Schultypen in Erfurt und Umgebung. In diesem Forschungsabschnitt finden Leitfadeninterviews statt (vgl. z.B. Flick 2016, Lamnek-Krell 2016). Die im Leitfaden enthaltenen Themen dienen einer (geringfügigen) Strukturierung des Gesprächs, im Unterschied etwa zum völlig offenen Konzept eines narrativen Interviews, in denen lediglich ein Erzählanreiz gegeben wird. Der Leitfaden enthält grob thematisch folgende zentrale Punkte: (a) Allgemeine Situation während des Homeschooling und der darauffolgenden Zeit, (b) Kommunikation und Erreichbarkeit, (c) Ausstattung mit digitalen (oder analogen) Lehr- und Lernmaterialien und Endgeräten, (d) Ausstattung mit speziell für DaZ und für den sprachsensiblen Unterricht notwendigen Materialien, (e) fachliches und didaktisches Know-how der Lehrkräfte hinsichtlich DaZ und sprachsensiblen Unterrichts und schließlich (f) Probleme, Desiderate und Wünsche bezüglich der Unterstützungsmaßnahmen. Es ist davon auszugehen, dass Lehrer(innen) über ein gutes Artikulationsvermögen verfügen und mit Interviewsituationen vertraut sind. Bei Schüler(inne)n, die Deutsch nicht als Erstsprache sprechen, ist das eher nicht vorauszusetzen. In solchen Fällen ist im qualitativen Forschungsparadigma entweder eine langwierige Interviewerschulung von Drittpersonen - Studierenden, Doktorand(innen) - notwendig oder erfahrene Forscher(innen) selbst führen die Gespräche. Deshalb werden die Interviews im Vorhaben von den Verfasser(inne)n, die bereits Forschungserfahrungen mit qualitativen Interviews im mehrsprachigen Kontext haben, persönlich durchgeführt. Die Interviews sind als Einzelgespräche unter Beachtung der jeweils geltenden Abstands- und Hygieneregeln sowie der datenschutzrechtlichen Bestimmungen vorgesehen. Sie werden auf einen digitalen Datenträger aufgezeichnet und anschließend inhaltsanalytisch ausgewertet. Hierzu bietet sich der inhaltlich-reduktive Ansatz an (im Sinne von Lamnek-Krell 2016). Im Anschluss an die qualitative Befragung, die mit relativ geringen Fallzahlen stattfindet, folgt eine standardisierte oder halbstandardisierte Online-Befragung (zur Methodik vgl. Atteslander 2010) unter Lehrer(inne)n, die aufgrund der Ergebnisse der ersten Interviews spezifischer und handlungsorientierter (hinsichtlich Unterstützungs- und Materialentwicklungsbedarf) gestaltet wird. Bei Schüler(innen) bleibt bei eventuell vertiefenden Befragungen der qualitative Untersuchungsansatz erhalten, da standardisierte Befragungen in dieser Zielgruppe an sprachlichen Hürden scheitern können und ohne zusätzliche Unterstützung erfahrungsgemäß zu Missverständnissen führen. Auf der Grundlage der empirischen Befunde werden im zweiten Schritt Lehrformate und Materialien entwickelt, die Lehrkräfte und Schüler(innen) in sprachlich und kulturell heterogenen Klassen in der Pandemiesituation, aber perspektivisch auch darüber hinaus, unterstützen. Hierbei handelt es sich um Beratungs- und Fortbildungsformate und didaktisches Material für Lehrende in den Bereichen DaZ, Sprachförderung und sprachsensibler Unterricht (vgl. hierzu generell Michalak-Lemke-Goeke 2015). Auf dem Gebiet sprachsensibler Unterricht und sprachsensible Unterrichtsmaterialien hat sich in den letzten Jahren einiges getan (vgl. z.B. Leisen 2016, Oleschko 2014 und auch online verfügbare 
didaktische Einheiten wie etwa beim Mercator Institut ${ }^{21}$ ). Die in diesen Projekten erzielten Ergebnisse bieten bestimmt eine gute allgemeine Basis; unser Projekt möchte jedoch spezifisch auf der Grundlage der empirisch ermittelten Bedarfe vor Ort und ausgehend von der aktuellen Situation in Thüringen Materialien erarbeiten. Ein weiteres besonderes Merkmal des Projekts besteht darin, dass unsere entwickelten Formate kontinuierlich, je nach Situation qualitativ oder quantitativ evaluiert werden.

\subsection{Universitärer Bearbeitungskontext des Projekts, Synergieeffekte und gesellschaftliche Relevanz}

Das Forschungsvorhaben ist an der Universität Erfurt angesiedelt, die eine primär geistes- und sozialwissenschaftlich ausgerichtete forschungsstarke Universität ist. Ihr Profil spiegelt sich u.a. im Querschnitts- und Forschungsfeld „Bildung. Schule. Verhalten“. Die Lehrerbildung hat sich in den letzten Jahrzehnten zu einem der profilgebenden Bereiche entwickelt; die Studierendenzahlen steigen in den Lehramtsstudiengängen kontinuierlich. Organisiert und geleitet wird die Lehrerbildung seit 2006 durch eine spezielle interfakultäre Einrichtung, die „Erfurt School of Education (ESE), Zentrum für Lehrerbildung und Bildungsforschung". Die Universität Erfurt beteiligt sich in der vom Bundesministerium für Bildung und Forschung (BMBF) drittmittelfinanzierten „Qualitätsoffensive Lehrerbildung“: Unter den Leitbegriffen „Identität, Immersion und Inklusion“ widmen sich im Gesamtvorhaben „QUALITEACH“ mehrere Teilprojekte der innovativen Weiterentwicklung der Erfurter Lehrerbildung. Eines dieser Teilprojekte ist das von den Verfasserinnen und dem Verfasser dieses Aufsatzes bearbeitete Projekt „Sprachliche Bildung in mehrsprachigen Kontexten". Innerhalb des Gesamtvorhabens ist es strukturell ein Teil des „Kompetenz- und Entwicklungszentrums für Inklusion in der Lehrerbildung": Es setzt sich auf verschiedenen Ebenen mit der Heterogenitätsdimension Mehrsprachigkeit auseinander. Auf der unterrichtsbezogenen Ebene soll als Projektertrag z.B. ein Pool an sprachsensiblen Lehr-Lern-Materialien entwickelt, erprobt und dauerhaft Studierenden wie Lehrenden zur Verfügung gestellt werden. Außerdem soll der sprachsensible Fachunterricht als Themenbereich in Kooperationen mit Vertreter(inne)n der Fachwissenschaften, Fachdidaktiken und Bildungswissenschaften auch auf hochschuldidaktischer Ebene verankert werden. Die Zielsetzungen dieses bereits laufenden und des in diesem Beitrag vorgestellten Projektes sind z.T. ähnlich, die Zielgruppen unterscheiden sich jedoch substanziell. Im bereits laufenden Projekt geht es um Studierende und Lehrende der Universität, im beantragten Projekt hingegen um Lehrer(innen) im Schuldienst und um Schüler(innen). Die interfakultäre Zusammenarbeit zwischen der Philosophischen Fakultät - vertreten durch das oben beschriebene Projekt „Sprachliche Bildung in mehrsprachigen Kontexten“ und die „Forschungsstelle für Interkulturalität und Mehrsprachigkeit" (FIM), am Lehrstuhl für Germanistische Sprachwissenschaft einerseits und der Erziehungswissenschaftlichen Fakultät (hier insbesondere das Großprojekt QUALITEACH) andererseits generiert eine produktive Kombination

21 https://www.mercator-institut-sprachfoerderung.de/de/publikationen/material-fuer-die-praxis/ methodenpool/. Abgerufen am 09.07.2020. 
linguistischer und erziehungswissenschaftlicher Zugänge und Methoden, was zu Synergieeffekten führt. So ist z.B. konkret für die erste explorative Projektphase der Feldzugang für die empirische Untersuchung zu Schulen und Lehrkräften gesichert. Der fachliche Austausch zwischen Fachwissenschaften, Fachdidaktiken und Bildungswissenschaften ermöglicht Konzepte von Team-Teaching im Bereich DaZ und den jeweiligen Fachdidaktiken. Ein weiteres dezidiertes Anliegen ist die Einbindung aller drei Phasen der Lehrerbildung, ${ }^{22}$ wie dies z.B. vom BMBF in der bundesweiten Qualitätsoffensive Lehrerbildung gefordert wird. Dies lässt sich durch die vorgesehenen Synergieeffekte folgendermaßen realisieren: (1) Die erste Phase der Lehrerbildung (also die universitäre Lehrerausbildung) ist durch unser laufendes oben beschriebenes Projekt vertreten. (2) Die zweite Phase der Lehrerbildung (d.h. der Vorbereitungsdienst) ist durch das seit dem Wintersemester 2016/17 existierende Zertifikatsprogramm „Deutsch als Fremdsprache/Deutsch als Zweitsprache für Lehrende“, das am Lehrstuhl für Germanistische Sprachwissenschaft entwickelt wurde (detailliert Földes 2017) beteiligt: Absolvent(inn)en dieses 30 Leistungspunkte (ECTS) umfassenden Studiums, die anschließend direkt ins Referendariat gehen bzw. Ausbildner(innen), die ebenfalls an diesem Programm teilnehmen, könnten im Wintersemester 2020/21 in Form von Schwerpunktseminaren und Praktika ins Projekt eingebunden werden und erste Ergebnisse direkt in ihre weitere Ausbildungs- und spätere Unterrichtspraxis transferieren. (3) Die dritte Phase der Lehrerbildung (d.h. die Fortbildung im Schuldienst) ist durch die im hier vorgestellten Vorhaben zu entwickelnden nachhaltigen Lehr- bzw. Lernformate für praktizierende Lehrer(innen) - über die Pandemie hinaus - mit involviert. Ein weiteres wichtiges Anliegen ist die Förderung des wissenschaftlichen Nachwuchses. Deshalb wurden im neuen Projekt eine Promotionsstelle und mehrere wissenschaftliche Hilfskraftstellen beantragt. Hierzu werden Master of Education-Studierende ausgewählt, die durch ihre Mitarbeit Projekterfahrungen in der empirischen Forschung und in der digitalen Materialentwicklung in DaZ sammeln und für ihre wissenschaftliche oder schulische Laufbahn nutzen können. Durch die Veröffentlichung der Unterrichtsmaterialien in einer Datenbank und in digitalen Lehr- bzw. Lernformaten wird die dauerhafte Verwendbarkeit der Projekterträge gewährleistet. Die Projektergebnisse sollen außerdem auf Tagungen und in Publikationen veröffentlicht werden und so über regionale Impulse hinaus die themenbezogene Forschung bereichern. Unser Projektantrag wurde auf die Sonderausschreibung der Volkswagen-Stiftung „Corona Crisis and Beyond - Perspectives für Science, Scholarship and Society" im Juni 2020 (in englischer Sprache) eingereicht. Die Verfasserinnen und der Verfasser möchten nun hoffen, dass das anvisierte Vorhaben bewilligt wird und zur weiteren Strukturentwicklung und Profilierung auch der Lehrerbildung an der Universität Erfurt beitragen kann.

22 In Deutschland besteht die Ausbildung von Lehrer(inne)n aus drei Phasen. Die erste beinhaltet die universitäre Ausbildung, die zweite ist der darauffolgende „Vorbereitungsdienst“ (auch Referendariat genannt) und als dritte Phase wird die regelmäßige Fortbildung von praktizierenden Lehrkräften betrachtet. 


\section{Ausblick}

Die Themen Bildungsgerechtigkeit, Bildungschancen und die damit einhergehende gesellschaftliche Teilhabe sind in einer pluralistischen Gesellschaft gewiss nicht neu, allerdings stellen sie sich im Lichte der aktuellen Situation neu. Wird sich die bereits in mancherlei Hinsicht bestehende Bildungsbenachteiligung für Schüler(innen) mit Migrationshintergrund weiter verschärfen oder gelingt es durch gezielte Maßnahmen den negativen Entwicklungen entgegenzusteuern? Das geplante Projekt möchte durch empirische Forschung und Entwicklung zur positiven Lösung des Problems einen innovativen Beitrag leisten.

\section{Literaturverzeichnis}

Aguado, Karin (2015): Triangulation: Möglichkeiten, Grenzen, Desiderate. In Triangulation in der Fremdsprachenforschung. Elsner, Daniela-Viebrock, Britta (Hrsg.). Frankfurt am Main: Peter Lang, 203-220. p.

Ahrenholz, Bernt (2017): Erstsprache - Zweitsprache Fremdsprache - Mehrsprachigkeit. In Deutsch als Zweitsprache. 4., vollständig überarbeitete und erweiterte Auflage. Ahrenholz, Bernt-Oomen-Welke, Ingelore (Hrsg.). Baltmannsweiler: Schneider Hohengehren, 3-20. p.

Atteslander, Peter (2010): Methoden der empirischen Sozialforschung. 13., neu bearbeitete Auflage. Berlin: Schmidt.

Baumann, Barbara (2017): Sprachförderung und Deutsch als Zweitsprache in der Lehrerbildung - ein deutschlandweiter Überblick. In Deutsch als Zweitsprache in der Lehrerbildung. Becker-Mrotzek, Michael-Rosenberg, Peter-Schroeder, Christoph-Witte, Annika (Hrsg.). Münster-New York: Waxmann, 9-26. p.

Becker-Mrotzek, Michael-Rosenberg, Peter-Schroeder, Christoph-Witte, Annika (Hrsg.) (2017): Deutsch als Zweitsprache in der Lehrerbildung. Münster-New York: Waxmann.

Benholz, Claudia-Frank, Magnus-Gürsoy, Erkan (Hrsg.) (2015): Deutsch als Zweitsprache in allen Fächern. Konzepte für Lehrerbildung und Unterricht. Stuttgart: Fillibach bei Klett.

Blok, Henk-Karsten, Sjoerd (2011): Inspection of Home Education in European Countries. European Journal of Education 46/1, 138-152. p.

Börsel, Anke-Lütke, Beate (2017): Deutsch als Zweitsprache in der Berliner Lehrkräftebildung. In Deutsch als Zweitsprache in der Lehrerbildung. Becker-Mrotzek, MichaelRosenberg, Peter-Schroeder, Christoph-Witte, Annika (Hrsg.). Münster-New York: Waxmann, 37-49. p.

Brabant, Christine (2008): Home Schooling. In The Routledge International Encyclopedia of Education. McCulloch, Gary-Crook, David (Hrsg.). London-New York: Routledge, 297-298. p.

Carlson, Sonja A.-Ehmke, Timo-Fischer, Nele-Hammer, Svenja-Köker, Anne-Ohm, Udo-Rosenbock-Agyei, Sonja (2018): DaZKom - a Structure Model of Pre-service Teachers' Competency for Teaching German as a Second Language in the Mainstream Classroom. In Professionelle Kompetenzen angehender Lehrkräfte im Bereich Deutsch als Zeitsprache. Ehmke, Timo-Hammer, Svenja-Köker, Anne-Ohm, Udo-Koch-Priewe, 
Barbara (Hrsg.). Münster-New York: Waxmann, 263-283. p.

Flick, Uwe (2016): Qualitative Sozialforschung. Eine Einführung. Vollständig überarbeitete und erweiterte Neuausgabe. 7. Auflage. Reinbek bei Hamburg: Rowohlt.

Földes, Csaba (2017): Interkulturalität - Heterogenität - Migration und ihre Folgen für Deutsch als Fremdsprache/Zweitsprache. Notizen aus einer Erfurter Perspektive. In Konstanz und Variation. Die deutsche Sprache in Mittel- und Ost- und Südosteuropa. Lăzărescu, loan-Sava, Doris (Hrsg.). Berlin: wvb Wissenschaftlicher Verlag Berlin, 106-124. p.

Gaither, Milton (2017): Homeschool. An American History. Revised Second Edition. New York: Palgrave Macmillan.

Michalak, Magdalena-Lemke, Valerie-Goeke, Marius (2015): Sprache im Fachunterricht. Eine Einführung in Deutsch als Zweitsprache und sprachbewussten Unterricht. Tübingen: Narr Francke Attempto.

Ladenthin, Volker (2018): Homeschooling. In Handbuch. Bildungsreform und Reformpädagogik. Barz, Heiner (Hrsg.). Wiesbaden: Springer VS, 519-525. p.

Lamnek, Siegfried-Krell, Claudia (2016): Qualitative Sozialforschung. 6., überarbeitete Auflage. Weinheim-Basel: Julius Beltz Verlag.

Leisen, Josef (2016): Handbuch Sprachförderung im Fach. Sprachsensibler Fachunterricht in der Praxis. Stuttgart: Klett.

Martin-Chang, Sandra-Levesque, Kyle (2017): Academic Achievement. Making an Informed Choice about Homeschooling. In The Wiley Handbook of Home Education. Gaither, Milton (Hrsg.). Chichester, UK-Malden, MA: Wiley Blackwell, 121-134. p.

Oleschko, Sven (2014): Zur Bedeutung der Sprache im gesellschaftswissenschaftlichen Lernprozess. In Sprache als Lernmedium im Fachunterricht. Theorien und Modelle für das sprachbewusste Lehren und Lernen. Michalak, Magdalena (Hrsg.). Baltmannsweiler: Schneider Hohengehren, 134-153. p.

Reimer, Franz (Hrsg.) (2012): Homeschooling. Bedrohung oder Bewährung des freiheitlichen Rechtsstaats? Baden-Baden: Nomos.

Rösch, Heidi (2009): Mitsprache. Deutsch als Zweitsprache - Sprachförderung in der Sekundarstufe 1. Grundlagen, Übungsideen. Braunschweig: Schrodel.

Spiegler, Thomas (2008): Home Education in Deutschland. Hintergründe - Praxis - Entwicklung. Wiesbaden: VS Verlag für Sozialwissenschaften.

Spiegler, Thomas (2012): Homeschooling in Deutschland und die Herausforderung seiner angemessenen rechtlichen Beurteilung. In Homeschooling. Bedrohung oder Bewährung des freiheitlichen Rechtsstaats? Reimer, Franz (Hrsg.). Baden-Baden: Nomos, 55-66. p.

Steinmüller, Ulrich (1984): Sprachstandserhebungen und Sprachförderung bei ausländischen Schülern der Sekundarstufe. Diskussion Deutsch 75, 77-90. p.

Wildemann, Anja-Hosenfeld, Ingmar (2020): Bundesweite Elternbefragung zu Homeschooling während der Covid 19-Pandemie. Erkenntnisse zur Umsetzung des Homeschoolings in Deutschland. Online unter https://www.uni-koblenz-landau.de/de/landau/fb5/bildung-kind-jugend/grupaed/medienordner-grundschulpaedagogik/Wildemann/bericht_homeschooling. Abgerufen am 07.07.2020. 